\section{\$30. 2-D MHD Simulation of Two Axially Colliding FRCs Accelerated by Magnetic Pressure Gradient}

Takahashi, T., Matsuzaki, K. (Gunma Univ.), Mizuguchi, N.

Field-Reversed Configurations (FRCs) are in extremely high beta state, and the high beta characteristic is attractive to be the core plasma of advanced fuel fusion. The FRC core plasma can be translated from the formation region, where the initial plasma is formed by the fieldreversed theta pinch method. The translated plasma is confined in a metal chamber, and it is built-up by such as the neutral beam injection. Recently, the C- 2 experiment by Tri-alpha energy Inc. extends dramatically the lifetime of FRC plasmas up to over 1 msec. ${ }^{1)}$ We simulate translation of FRC plasma by using the 2-D resistive MHD code and study properties of the translation motion of FRC accelerated by a time-varying external field; it controls the translation speed and colliding process. The resistivity in this study is assumed as a constant value here. And we analyze how the FRC core is accelerated by the timedependent external field and is subject to the subsequent axial collision with a counter FRC.

The resistive MHD simulation is carried out for the plasma formed on the NUCTE-III machine. To translate an FRC plasma in our simulation, the mirror field strength of the one axial side is weakened; this results in the straight field line along the radial boundary of the calculation region. Temporal change in the 2D magnetic flux profile is shown in Fig. 1. It is found that the FRC plasma is expanded axially as it moves toward the right in Fig. 1. This calculation shows that the separatrix length increases from $0.4 \mathrm{~m}$ to $0.9 \mathrm{~m}$. On the other hand, the radius shrinks from about $0.1 \mathrm{~m}$ to $0.06 \mathrm{~m}$. Our simulation successfully shows the FRC translation process.

Next, we present the MHD simulation result for axially colliding phenomena of two FRCs accelerated by both the magnetic pressure gradient and the magnetic pusher that compresses the plasma by axially travelling mirror field. Behavior of axially colliding FRCs is presented in Fig. 2. The separatrix shapes are strongly deformed by axial force from the magnetic pusher at $21.8 \mu \mathrm{s}$. And then, the FRC plasmas decay gradually at the same location by resistive dissipation. We find that at $65.3 \mu \mathrm{s}$ the two separatrices are connected to each other, and the FRC plasmas become a doublet that has two peaks of trapped flux inside one separatrix surface. The outermost field-line, however, still has curvature at $65.3 \mu \mathrm{s}$ and it will become straight by the magnetic tension force.

Since the resistivity in our simulation model has been assumed to be a constant value, FRC merging never be observed in our simulation. However, the two separatrices probably connects to each other, and the typical phenomena observed in the magnetic reconnection have been found here, such as peaking of the current density. Effects of the

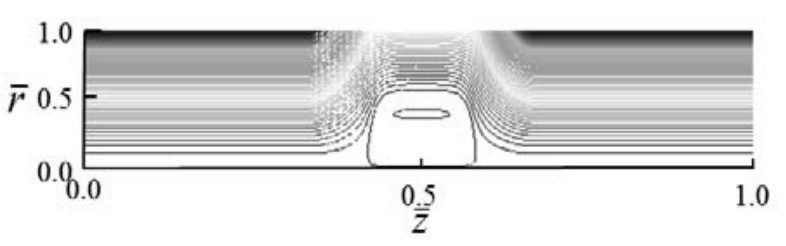

(a) Initial position of a FRC plasma

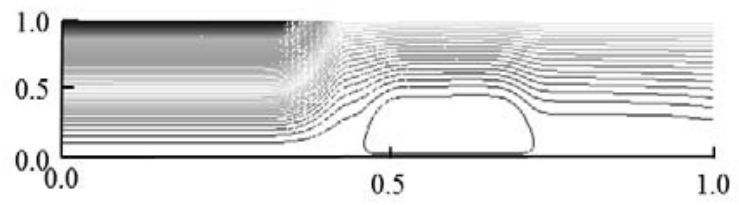

(b) $t=29.0 \mu \mathrm{s}$

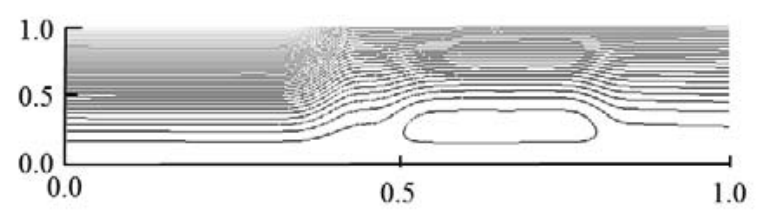

(c) $t=58.1 \mu \mathrm{s}$

Fig. 1. Temporal change in the 2D magnetic flux profile.

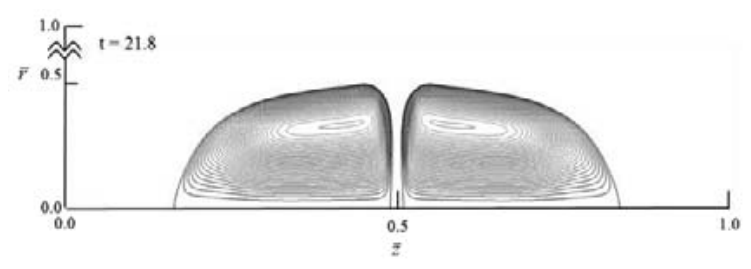

(a) $t=21.8 \mu \mathrm{s}$

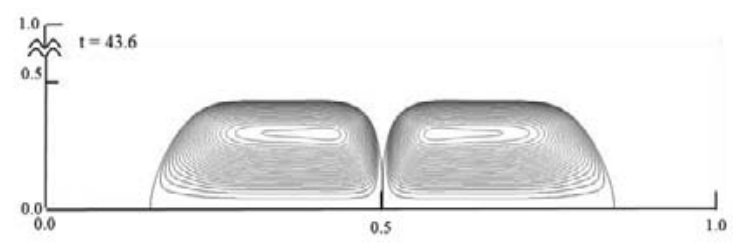

(b) $t=43.6 \mu \mathrm{s}$

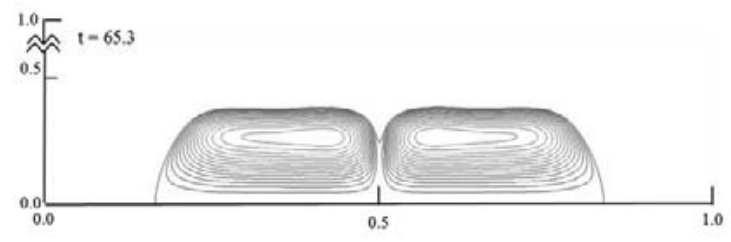

(c) $t=65.3 \mu \mathrm{s}$

Fig. 2. Magnetic flux profile of axial collision of two FRCs

resistivity on the collision and merging process will be studied in near future.

1) Binderbauer, M. W. et al.: Phys. Rev. Lett. 105, 0450031-4 (2010). 